\section{Fatores associados à displasia broncopulmonar em prematuros sob ventilação mecânica precoce}

\section{Factors associated with bronchopulmonary dysplasia in premature infants under early mechanical ventilation}

Paula Eylla Cristina Rodrigues Duarte 1

Sônia Bechara Coutinho 2

\begin{abstract}
Objectives: Objectives: to describe the neonatal and assisted ventilation factors associated with bronchopulmonary dysplasia (BPD) and verify their frequency in premature newborns undergoing mechanical ventilation (MV) in the first week of life.

Methods: retrospective cohort study carried out at the Neonatal Intensive Care Unit. The medical records of 86 premature infants under $M V$ in the first week of life were analyzed and neonatal data, $M V$ parameters and their relationship with BPD registered. To verify the association between the variables of the study and BPD, the chi-square test and the Fisher exact test were used as appropriate. The t-test and Kruskal Wallis test were used to compare the means of the continuous variables.

Results: BPD occurred in $17.4 \%$ of cases. Factors related to the illness were: lower birth weight and gestational age, Apgar $<7$ at 1 and 5 minutes, greater time under antibiotic therapy, parenteral nutrition and $M V$, higher values of the fraction of inspired oxygen $\left(\mathrm{FiO}_{2}\right), \mathrm{MV}$ as the first respiratory aid, lower volume of enteral nutrition and ponderal gain. No difference was found in the level of positive inspiratory pressure, end positive expiratory pressure and pressure difference.

Conclusions: the occurrence of BPD was low and related to clinical and nutritional management and early, prolonged MV. With the exception of mean $\mathrm{FiO}_{2}$, no relationship was found between the illness and ventilation parameters.
\end{abstract}

Key words Infant, premature, Respiration, artificial, Oxygen inhalation therapy, Bronchopulmonary dysplasia
1,2 Pós-graduação em Saúde da Criança e do Adolescente. Centro de Ciências da Saúde. Universidade Federal de Pernambuco. Av. Professor Moraes Rego, 1235. Cidade Universitária. Recife, PE, Brasil. CEP: 50.670-901. E-mail: paulinha_duarte@yahoo.com.br

\section{Resumo}

Objetivos: descrever os fatores neonatais e de assistência ventilatória associados à displasia broncopulmonar (DBP), e verificar sua frequência em recém-nascidos prematuros submetidos à ventilação mecânica (VM) na primeira semana de vida.

Métodos: coorte retrospectiva, realizada em Unidade de Terapia Intensiva Neonatal. Foram analisados prontuários de 86 prematuros, sob VM na primeira semana de vida e registrados dados neonatais, parâmetros da VM e sua relação com a $D B P$. Para verificar a associação entre as variáveis do estudo e a DBP utilizou-se o teste do qui-quadrado e o Exato de Fisher quando indicado. O teste te o Kruskal Wallis foram utilizados para a comparação das médias das variáveis contínuas.

Resultados: a DBP ocorreu em 17,4\%. Foram relacionados à doença: menor peso ao nascer e idade gestacional, Apgar $<7$ no $1^{\circ}$ e $5^{\circ}$ minutos, maior tempo sob antibioticoterapia, nutrição parenteral e $V M$, valores elevados de fração inspirada de oxigênio $\left(\mathrm{FiO}_{2}\right), V M$ como primeiro suporte respiratório, menor volume de nutrição enteral e ganho ponderal . Não houve diferença nos niveis de pressão positiva inspiratória, pressão positiva expiratória final e diferença de pressão.

Conclusões: a ocorrência da DBP foi baixa e relacionada ao manejo clínico e nutricional e VM precoce e prolongada. Excetuando-se a $\mathrm{FiO}_{2}$ média não foi encontrada relação entre a doença e os demais parâmetros ventilatórios.

Palavras-chave Prematuro, Respiração artificial, Oxigenoterapia, Displasia broncopulmonar 


\section{Introdução}

Os progressos na assistência perinatal e neonatal nas últimas décadas têm aumentado a sobrevida de recém-nascidos com pesos de nascimento e idades gestacionais cada vez menores, em uma fase precoce do seu desenvolvimento pulmonar. A ocorrência de morbidades na evolução desses neonatos é frequente, sendo uma delas a Displasia Broncopulmonar (DBP), considerada uma das complicações crônicas mais importantes em prematuros sobreviventes. 1,2

Há uma relação inversa entre incidência da DBP com a idade gestacional e o peso ao nascimento da criança. ${ }^{1,3} \mathrm{Em}$ estudo multicêntrico realizado em países da America Latina com recém-nascidos entre 500 a 1500 gramas, a incidência da DBP foi $24,4 \%$. 1 No Brasil, em pesquisas recentes a incidência variou entre $21 \%$ a $68 \%$.3-7 Em Pernambuco, uma pesquisa realizada em maternidade de referência, a incidência de DBP foi $17,6 \%$ entre recém-nascidos prematuros e com muito baixo peso. ${ }^{8}$ Esta variação pode ser atribuída a heterogeneidade das populações e dos cuidados aos neonatos 9 além das diferentes definições utilizadas para a doença entre os estudos.

Atualmente, a DBP é definida por Jobe e Bancalari10 como a necessidade de oxigênio suplementar acima de $21 \%$ por um período igual ou maior a 28 dias. É considerada uma doença multifatorial, associada ao parto prematuro e a eventos como inflamação/infecção pré e pós-natais, baixo peso ao nascer, síndrome do desconforto respiratório (SDR), persistência do canal arterial, fatores nutricionais e principalmente pela exposição prolongada à ventilação mecânica (VM) e ao oxigênio.1,9,11-14

Apesar de bem estabelecido o papel da VM e do oxigênio no desenvolvimento da DBP, ainda são escassas as publicações que estudam a relação de seu emprego nos primeiros dias de vida e/ou os parâmetros ventilatórios e de concentração de oxigênio utilizados com o desenvolvimento da doença.3,5,11,15,16 Algumas destas pesquisas que analisaram prematuros submetidos à VM, demonstraram relação entre o emprego de valores de pressão inspiratória positiva (PIP) acima de 20 $\mathrm{cmH}_{2} \mathrm{O}$ e DBP, bem como a administração de uma concentração de oxigênio média de 0,62 e o desenvolvimento da doença. $3,11,16$ A precocidade da instituição da VM também parece estar relacionada à doença, conforme pesquisa realizada por Van Marter et al. 15 o emprego da VM no primeiro dia de vida foi o único fator de risco associado à DBP. Segundo Bhering et al. 5 recém-nascidos prematuros e com necessidade de VM por mais de dois dias têm $54,4 \%$ de chance de desenvolver a doença.

Contudo, alguns aspectos devem ser considerados nestas pesquisas: a seleção da população analisada é composta apenas de crianças com muito baixo peso ao nascer, ou seja, aquelas muito prematuras com maior chance de desenvolver a doença e há apenas um estudo brasileiro ${ }^{3}$ que investigou os parâmetros utilizados em crianças com e sem DBP submetidas à VM.

Com base nessa premissa, este artigo teve por objetivos descrever os fatores neonatais e de assistência ventilatória associados à DBP, além de verificar sua frequência em recém-nascidos prematuros ventilados na primeira semana de vida em uma unidade neonatal.

\section{Métodos}

Estudo de coorte retrospectivo, realizado na Unidade de Terapia Intensiva Neonatal (UTIN) do Hospital das Clínicas, vinculado à Universidade Federal de Pernambuco, em Recife.

Os dados foram coletados de janeiro de 2006 a dezembro de 2008, através da consulta direta ao banco de dados da UTIN e dos prontuários dos pacientes no serviço de arquivos médicos da instituição.

O tamanho da amostra foi determinado pelo período de observação tendo sido um pouco inferior ao estimado ( $\mathrm{n}=97)$, considerando uma frequência esperada da DBP de $18 \%$ (baseada no trabalho de Lima et al. ${ }^{8}$ ), um erro máximo tolerável de $5 \%$ e um poder de $80 \%$.

Foram incluídos todos os recém-nascidos com idade gestacional inferior a 37 semanas submetidos à VM convencional na primeira semana de vida. Recém-nascidos com malformações congênitas graves, síndromes genéticas, transferidos ou que faleceram antes do $28^{\circ}$ dia de vida, foram excluídos da pesquisa.

A DBP foi definida como necessidade de uso contínuo de oxigênio suplementar acima de $21 \%$ até o $28^{\circ}$ dia de vida. 10 Segundo Jobe e Bancalari 10 a administração de oxigênio nos primeiros 28 dias de vida resultou em alta sensibilidade, especificidade e valor preditivo positivo e negativo para administração de oxigênio com 36 semanas de idade pósmenstrual em prematuros. Além disto, o registro de dados até o $28^{\circ}$ dia de vida para classificação quanto à ocorrência de DBP permitiu incluir crianças com tempo de exposições semelhantes aos fatores pesquisados tornando, portanto, a amostra mais homogênea.

A ventilação mecânica convencional foi consi- 
derada aquela com pressão positiva intermitente gerada por ventiladores com fluxo contínuo, ciclados a tempo e limitados a pressão. Os ventiladores utilizados eram da marca Inter $3^{\circledR}$ (Intermed).

Foram analisadas as variáveis neonatais: idade gestacional (semanas), peso ao nascer (gramas), sexo, adequação peso ao nascer para a idade gestacional17 ${ }^{1}$ PIG $=$ neonatos com peso abaixo do percentil 10 e $\mathrm{AIG}=$ neonatos com peso entre os percentis 10 e 90 ), Apgar no $1^{\circ}$ e $5^{\circ}$ minutos, ventilação com balão auto-inflável em sala de parto (máscara facial ou intubação), síndrome do desconforto respiratório, uso do surfactante exógeno, dias de antibioticoterapia, nutrição parenteral (início e duração), volume de nutrição enteral (registrado em $\mathrm{mL} /$ dia e calculada a média do volume total recebido durante internação) e peso diário (mensurado pela equipe de enfermagem em balança eletrônica calibrada Filizola BP Baby ${ }^{\circledR}$ e registrado em gramas) o qual foi calculada média durante o período de internação pesquisado.

Em relação à assistência ventilatória foram analisadas as seguintes variáveis: tipo de suporte de oxigênio na admissão - primeiro suporte utilizado pelo neonato para oxigenoterapia: halo, pressão positiva contínua na via aérea nasal (CPAPn), ventilação com pressão positiva intermitente nasal (nIPPV) ou VM - início da VM $\left(1^{\circ}, 2^{\circ}, 3^{\circ}, 4^{\circ}, 5^{\circ}, 6^{\circ}\right.$ ou $7^{\circ}$ dia) tempo sob VM (total de dias). Foram registrados ainda, todos os níveis diários de pressão inspiratória positiva (PIP), pressão positiva expiratória final (PEEP) e diferença de pressão $(\Delta \mathrm{P})$ entre PIP e PEEP durante permanência do prematuro em VM, além dos valores diários de fração inspirada de oxigênio $\left(\mathrm{FiO}_{2}\right)$ independente do suporte utilizado para sua administração.

Após a coleta, os dados foram tabulados em planilha do software Excel (Microsoft) e para variáveis que tinham acompanhamento diário (PIP, PEEP, $\Delta \mathrm{P}, \mathrm{FiO}_{2}$, peso, uso de nutrição parenteral, uso de nutrição enteral e volume de nutrição enteral) eram calculadas suas respectivas médias.

Para variáveis contínuas utilizou-se média, desvio-padrão e intervalo de confiança para média. Para variáveis categóricas foram calculadas as respectivas frequências e a fim de verificar associação das variáveis do estudo com o desfecho pesquisado, foi empregado o teste do qui-quadrado para independência. Nos casos em que a frequência esperada foi menor que cinco em mais de $20 \%$ das caselas da tabela de contingência, foi utilizado o teste Exato de Fisher. Para realização do teste de comparação de médias utilizou-se o teste $\mathrm{t}$ de
Student e nas variáveis onde a suposição de normalidade não foi satisfeita, o teste não paramétrico de Kruskal Wallis. O software SPSS 13.0 para Windows foi usado na análise. Valores de $p<0,05$ foram considerados significantes.

O estudo foi aprovado pelo Comitê de Ética em Pesquisa do Centro Integrado de Saúde Amaury de Medeiros - PE.

\section{Resultados}

No período do estudo nasceram 938 crianças prematuras. Destas, 780 não utilizaram ou iniciaram VM após o sétimo dia de vida, 59 foram óbitos neonatais, 09 continham malformações congênitas, 03 apresentaram alguma síndrome genética e 01 foi transferida durante o período neonatal e foram excluídas da pesquisa.

Assim, 86 prematuros preencheram os critérios de inclusão e puderam ser analisados quanto ao desfecho. Evoluíram sem DBP $71(82,6 \%)$ das crianças e $15(17,4 \%)$ apresentaram a doença (Figura $1)$.

As médias de peso ao nascer e idade gestacional foram significativamente menores nas crianças com DBP conforme apresentado na Tabela 1. Ainda na mesma tabela, observa-se que prematuros com DBP apresentaram maior frequência de Apgar inferior a 7 no primeiro e quinto minutos quando comparados com aqueles sem DBP.

Na Tabela 2, em relação à assistência neonatal, verifica-se que neonatos com DBP utilizaram nutrição parenteral por período maior. Por outro lado, estas crianças receberam menor média de volume de nutrição enteral e apresentaram menor peso médio durante o período de internação, dados estatisticamente significantes. O número médio de dias em uso de antibiótico foi significativamente maior entre recém-nascidos com DBP.

Conforme dados sobre assistência ventilatória apresentados na Tabela 3, VM foi o suporte respiratório primariamente utilizado em $80 \%$ dos recémnascidos que desenvolveram DBP posteriormente. Aqueles sem DBP foram inicialmente submetidos a modos não invasivos de assistência ventilatória $(58,2 \%)$. O tempo de permanência em VM também alcançou diferença expressiva entre os grupos, com $66,7 \%$ dos neonatos que desenvolveram DBP e apenas $2,8 \%$ do outro grupo, ainda sob VM no $15^{\circ}$ dia de vida. Entre os parâmetros ventilatórios avaliados apenas a média de $\mathrm{FiO}_{2}$ foi maior no grupo DBP $(p=0,038)$. 
Figura 1

Diagrama dos recém-nascidos estudados entre janeiro de 2006 e dezembro de 2008 .

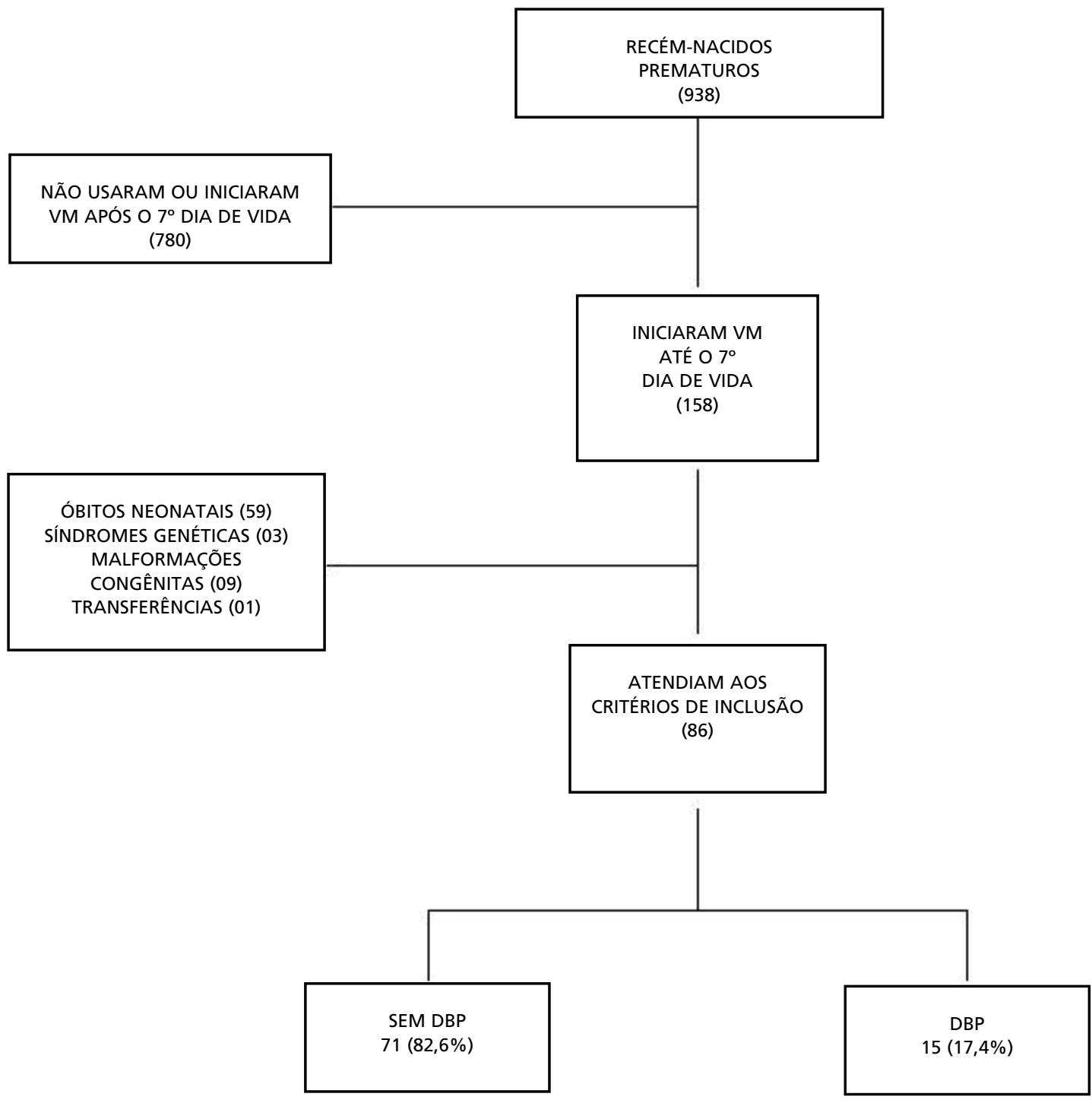


Características biológicas e doenças neonatais dos recém-nascidos ventilados com e sem displasia broncopulmonar na unidade de terapia intensiva neonatal. Hospital das Clínicas, PE, 2006-2008.

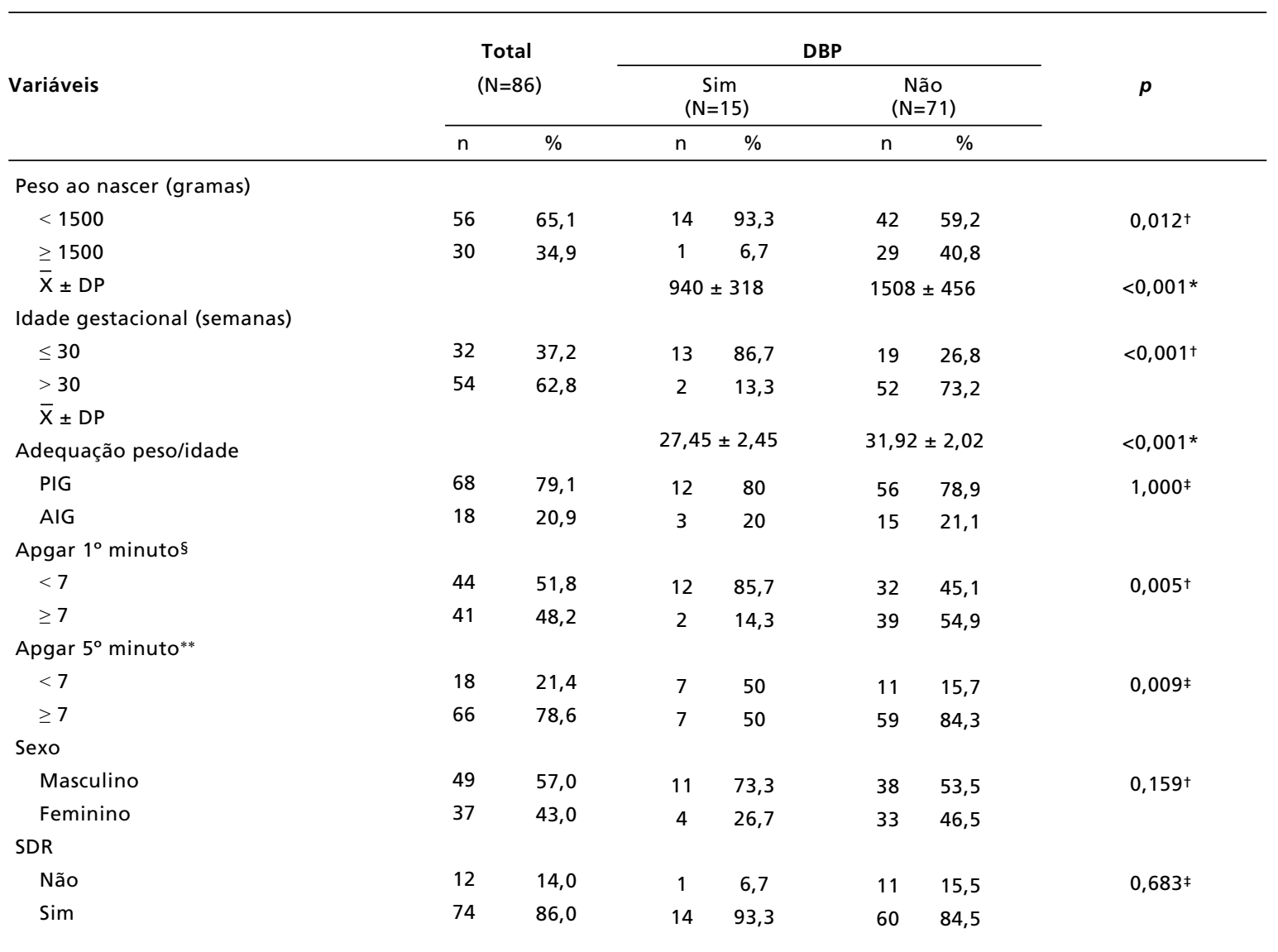

DBP = displasia broncopulmonar; PIG = pequeno para idade gestacional; $\mathrm{AIG}=$ adequado para a idade gestacional; SDR = síndrome do desconforto respiratório; *Teste de Kruskal Wallis; † Teste de qui-quadrado para independência; ₹ Teste exato de Fisher; §1 caso ignorado; ${ }^{* *} 2$ casos ignorados. 
Tabela 2

Assistência neonatal e evolução ponderal dos recém-nascidos ventilados com e sem displasia broncopulmonar na unidade de terapia intensiva neonatal. Hospital das Clínicas, PE, 2006-2008.

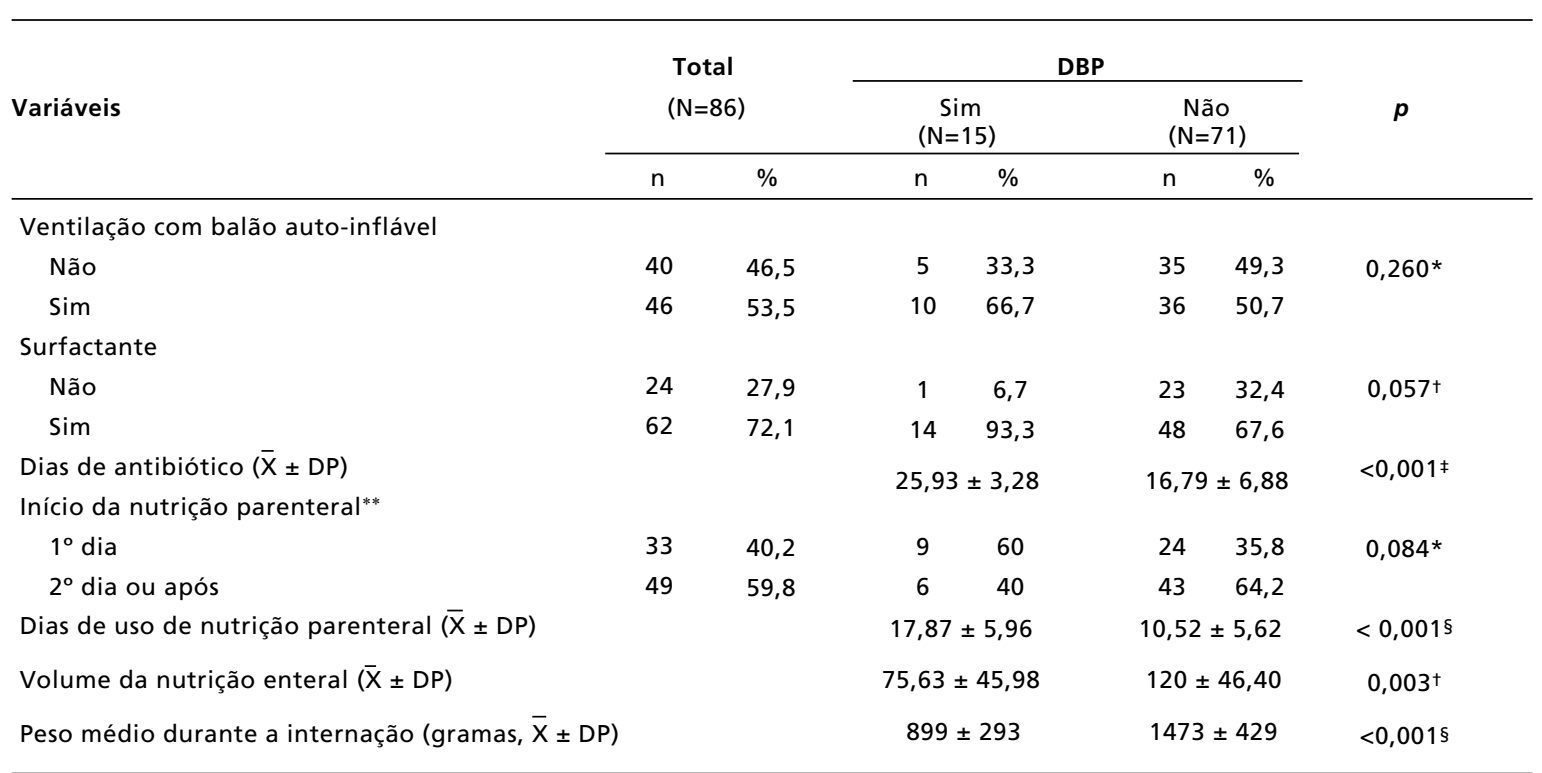

* Teste de qui-quadrado para independência; † Teste exato de Fisher; ${ }^{\ddagger}$ Teste de Kruskal Wallis; $§$ Teste t-student. ${ }^{* *} 6$ casos ignorados.

Tabela 3

Manejo da ventilação mecânica e do oxigênio dos recém-nascidos ventilados com e sem displasia broncopulmonar na unidade de terapia intensiva neonatal. Hospital das Clínicas, PE, 2006-2008.

\begin{tabular}{|c|c|c|c|c|c|c|c|}
\hline \multirow[b]{2}{*}{ Variáveis } & \multirow{2}{*}{\multicolumn{2}{|c|}{$\begin{array}{l}\text { Total } \\
(\mathrm{N}=86)\end{array}$}} & \multicolumn{4}{|c|}{ DBP } & \multirow[b]{2}{*}{$p$} \\
\hline & & & \multicolumn{2}{|c|}{$\operatorname{Sim}_{(N=15)}$} & \multicolumn{2}{|c|}{$\begin{array}{c}\text { Não } \\
(\mathrm{N}=71)\end{array}$} & \\
\hline \multicolumn{8}{|c|}{ Suporte de $\mathrm{O}_{2}$ na admissão** } \\
\hline CPAPn/nIPPV & 42 & 51,2 & 3 & 20 & 39 & 58,2 & 0,007 * \\
\hline \multicolumn{8}{|l|}{ Início da VM } \\
\hline $1^{\circ} \mathrm{dia}$ & 66 & 76,8 & 14 & 93,3 & 52 & 73,2 & $0,175^{\dagger}$ \\
\hline $2^{\circ}$ dia ou após & 20 & 23,2 & 1 & 6,7 & 19 & 26,8 & \\
\hline \multicolumn{8}{|c|}{ Tempo de uso da VM (dias) } \\
\hline Até 7 & 56 & 65,1 & 2 & 13,3 & 54 & 76,1 & $<0,001^{\dagger}$ \\
\hline \multicolumn{8}{|l|}{$\mathrm{PIP}\left(\mathrm{cmH}_{2} \mathrm{O}\right)$} \\
\hline$\leq 15$ & 34 & 39,5 & 4 & 26,7 & 30 & 42,2 & $0,262 *$ \\
\hline$>15$ & 52 & 60,5 & 11 & 73,3 & 41 & 57,8 & \\
\hline $\bar{x} \pm D P$ & & & \multicolumn{2}{|c|}{$16,22 \pm 2,10$} & \multicolumn{2}{|c|}{$16,02 \pm 2,88$} & $0,760 \S$ \\
\hline \multicolumn{8}{|l|}{ PEEP $\left(\mathrm{cmH}_{2} \mathrm{O}\right)$} \\
\hline$<5$ & 76 & 88,4 & 13 & 86,7 & 63 & 88,7 & $1,000^{+}$ \\
\hline$\geq 5$ & 10 & 11,6 & 2 & 13,3 & 8 & 11,3 & \\
\hline $\bar{X} \pm D P$ & & & \multicolumn{2}{|c|}{$4,57 \pm 0,25$} & \multicolumn{2}{|c|}{$4,47 \pm 0,35$} & $0,205 \S$ \\
\hline
\end{tabular}


Manejo da ventilação mecânica e do oxigênio dos recém-nascidos ventilados com e sem displasia broncopulmonar na unidade de terapia intensiva neonatal. Hospital das Clínicas, PE, 2006-2008.

\begin{tabular}{|c|c|c|c|c|c|c|c|}
\hline \multirow{3}{*}{ Variáveis } & \multirow{2}{*}{\multicolumn{2}{|c|}{$\begin{array}{l}\text { Total } \\
(\mathrm{N}=86)\end{array}$}} & \multicolumn{4}{|c|}{ DBP } & \multirow{3}{*}{$p$} \\
\hline & & & \multicolumn{2}{|c|}{$\operatorname{Sim}_{(N=15)}$} & \multicolumn{2}{|c|}{$\begin{array}{c}\text { Não } \\
(\mathrm{N}=71)\end{array}$} & \\
\hline & $\mathrm{n}$ & $\%$ & $\mathrm{n}$ & $\%$ & $\mathrm{n}$ & $\%$ & \\
\hline \multicolumn{8}{|l|}{$\Delta \mathrm{P}\left(\mathrm{cmH}_{2} \mathrm{O}\right)$} \\
\hline$\leq 10$ & 20 & 23,3 & 1 & 6,7 & 19 & 26,8 & $0,175^{+}$ \\
\hline $\bar{X} \pm D P$ & & & \multicolumn{2}{|c|}{$11,91 \pm 1,73$} & \multicolumn{2}{|c|}{$11,46 \pm 2,63$} & $0,407 \S$ \\
\hline \multicolumn{8}{|l|}{$\mathrm{FiO}_{2}$} \\
\hline$\leq 0,4$ & 51 & 59,3 & 6 & 40 & 45 & 63,4 & $0,198^{\dagger}$ \\
\hline $0,45-0,5$ & 25 & 29,1 & 7 & 46,7 & 18 & 25,4 & \\
\hline$>0,5$ & 10 & 11,6 & 2 & 13,3 & 8 & 11,2 & \\
\hline
\end{tabular}

$\mathrm{VM}=$ ventilação mecânica; $\mathrm{DBP}=$ displasia broncopulmonar; $\mathrm{O}_{2}=$ oxigênio; CPAPn = pressão positiva contínua na via aérea nasal; nIPPV = ventilação com pressão positiva intermitente nasal; $\mathrm{PIP}=$ pressão inspiratória positiva; $\mathrm{cmH}_{2} \mathrm{O}=\mathrm{centímetros}$ de água: $\mathrm{PEEP}=$ pressão positiva expiratória final; $\Delta \mathrm{P}=$ diferença de pressão; $\mathrm{FiO}_{2}=$ fração inspirada de oxigênio; * Teste de quiquadrado para independência; † Teste exato de Fisher; ₹ Teste de Kruskal Wallis; § Teste t-student; ** 2 casos em halo e 2 em ar ambiente.

\section{Discussão}

$\mathrm{Na}$ amostra analisada, a frequência da DBP foi $17,4 \%$, inferior a relatada por Cunha et al. 3 que referiram uma incidência de $52,3 \%$ em crianças submetidas à VM na primeira semana de vida.

Como esperado, maior frequência da doença ocorreu entre crianças mais prematuras, com muito baixo peso ao nascer, que receberam VM por mais dias e $\mathrm{FiO}_{2}$ mais elevadas. Contrariando as expectativas, os parâmetros ventilatórios utilizados não obtiveram relação com o desfecho pesquisado.

Por se tratar de estudo com delineamento retrospectivo, o volume corrente administrado durante $\mathrm{VM}$, fator reconhecidamente associado à DBP, não foi avaliado devido à ausência de tecnologia adequada para mensuração no serviço. Além desta, outras variáveis ligadas a VM como o fluxo inspiratório e tempo inspiratório eram escassamente registrados e não foram incluídas na análise. Casos de canal arterial patente também não foram analisados, pois não havia confirmação diagnóstica através da realização do ecocardiograma em algumas crianças.

Outro fator limitante nesta pesquisa foi o número reduzido de doentes na amostra, que não recomendaria uma análise multivariada. Entretanto, em caráter exploratório a análise foi realizada (dados não apresentados), onde as variáveis tempo de uso da VM, idade gestacional e Apgar $5^{\circ}$ minuto apre- sentaram associação independente no modelo final, porém com intervalos de confiança amplos. Sugerimos a repetição desta análise em outros estudos com maior tamanho de amostra.

Nesta coorte, assim como em outras pesquisas 1,3 a frequência de DBP foi maior entre os recémnascidos com menor idade gestacional e peso ao nascimento. Seguindo a tendência na melhora dos cuidados ao neonato de risco, a maioria das crianças analisadas recebeu surfactante exógeno. Tal resultado, contudo era esperado por se tratar de uma amostra constituída exclusivamente de prematuros, com maior probabilidade de evoluir com SDR como observado nos resultados. Mas, assim como na publicação de Cunha et al. ${ }^{3}$ não houve associação entre SDR com a doença, provavelmente pela alta frequência de uso do surfactante nesta pesquisa.

O recém-nascido, especialmente o prematuro, pode encontrar dificuldade na transição do meio intra para o extra-uterino, sendo o boletim de Apgar utilizado para avaliar a resposta do neonato às manobras de reanimação e a eficácia das mesmas. ${ }^{18}$ No presente estudo, foi significativamente maior o percentual de crianças com Apgar inferior a 7 no primeiro e quinto minutos, resultado que diferiu daqueles encontrados em outras publicações. 3,4

A reanimação com ventilação manual, quando necessária, deve ser aplicada de forma criteriosa, pois, seu uso com alto volume pode causar injúria pulmonar, desencadeando processo inflamatório 
perpetuado pelo uso posterior da VM.19 Porém, não foi evidenciada relação desta prática com o desenvolvimento da doença na coorte analisada.

Outra característica do prematuro é estar mais suscetível ao aparecimento de infecções, condição onde há aumento no nível de mediadores inflamatórios. Estes, podem lesar o tecido pulmonar, colaborando na ocorrência de DBP, 1,20 sendo a administração de antibióticos necessária. A utilização de antibioticoterapia após o $5^{\circ}$ dia de vida foi associada ao aumento no risco de desenvolver DBP segundo resultados de Teixeira et al. ${ }^{7}$ No presente estudo, todas as crianças receberam antibioticoterapia, com uma média de dias expressivamente maior naqueles prematuros do grupo DBP.

O surgimento de infecções respiratórias e sepse podem ser favorecidos ainda, pela desnutrição, que reduz a força dos músculos respiratórios, levando à falência muscular, aumentando a necessidade e o tempo sob ventilação assistida. ${ }^{21} \mathrm{Na}$ presente pesquisa, a nutrição parenteral foi administrada precocemente e seu tempo de uso foi maior nas crianças com DBP. Embora esta prática seja necessária para a manutenção do suporte nutricional do pré-termo, existe aumento no risco de morte por sepse na fase neonatal tardia relacionada ao seu uso. 22

No que concerne à alimentação enteral, o volume administrado foi mais baixo naquelas crianças que evoluíram com DBP, bem como a média de peso alcançada durante sua permanência na UTIN, refletindo a dificuldade em alimentar o prematuro, que pode ter períodos de interrupção em sua alimentação por razões como imaturidade gastrintestinal, administração de indometacina, comprometimento respiratório ou instabilidade cardiovascular. ${ }^{13}$

$\mathrm{Na}$ assistência ventilatória ao neonato pré-termo a instituição de estratégias não invasivas, entre elas o uso precoce da CPAPn, vêm sendo associadas com a redução na incidência da DBP e na ativação de mediadores inflamatórios no pulmão. ${ }^{23,24}$ Contudo, nesta pesquisa, a VM foi o suporte inicialmente adotado em $80 \%$ das crianças do grupo DBP. No grupo sem a doença, entretanto, constatou-se a tentativa em instituir a ventilação não invasiva após o nascimento, porém $73,2 \%$ destas crianças necessitaram de VM logo no primeiro dia de vida. Segundo pesquisa realizada por Van Marter et al. 15 o uso precoce da VM foi o único fator de risco para DBP, aumentando em até 13 vezes a chance da doença naquelas crianças nas quais o suporte foi iniciado no primeiro dia de vida.

O número médio de dias em VM diferiu acentuadamente entre os grupos. O período de permanência foi quase quatro vezes maior nas crianças com DBP, resultado semelhante ao relatado por outros autores. ${ }^{1,11}$ Importante destacar que no décimo quinto dia de vida mais da metade dos prematuros do grupo DBP ainda permaneciam em $\mathrm{VM}$, e apenas $2,8 \%$ daqueles sem a doença ainda usavam o mesmo suporte.

Algumas publicações destacam a importância do uso precoce de estratégias de assistência respiratória protetoras durante a permanência em VM, pois a lesão pulmonar pode acontecer já nos primeiros ciclos respiratórios. 25,26 Os resultados encontrados sinalizam, que tais práticas parecem ser adotadas pelo serviço, com valores de PIP utilizados em torno de $16 \mathrm{cmH}_{2} \mathrm{O}$ para os dois grupos, inferiores aqueles descritos em outras publicações onde os níveis utilizados foram acima de $20 \mathrm{cmH}_{2} \mathrm{O} .3,11,16$ Apenas Cogo et al. 11 descrevem valor médio de PIP inferior ao relatado neste artigo $\left(13,9 \mathrm{cmH}_{2} \mathrm{O}\right)$ e apenas no grupo sem DBP.

A utilização de PEEP adequada exerce papel protetor durante a VM, estabilizando alvéolos, evitando a degradação de surfactante pulmonar e minimizado/retardando o início da lesão pulmonar induzida pela VM.25,27 Entretanto, assim como no estudo de Cogo et al. 11 os valores de PEEP utilizados na instituição pesquisada não diferiram entre os grupos, todavia os níveis médios empregados foram mais altos que os relatados pelos autores.

Apesar da injúria pulmonar advir da administração de volumes correntes elevados, na prática clínica nem sempre é possível monitorizar as pressões e volumes pulmonares nos recém-nascidos em VM, utilizando-se o valor de PIP como referência para evitar a hiperdistensão pulmonar. Desta forma, $\Delta \mathrm{P}$ entre a PIP e a PEEP, demonstra uma medida indireta do volume corrente, que nos neonatos é também influenciado pelas complacências pulmonar e da caixa torácica. ${ }^{28,29} \mathrm{Em}$ ambos os grupos estudados, os valores utilizados foram similares e sem diferença significativa entre os grupos. Contudo, as crianças que desenvolveram DBP eram mais prematuras, e provavelmente com tórax mais complacente, o que pode ter influenciado no desenvolvimento da doença.

É bem estabelecida na literatura, a estreita relação entre a ação lesiva do oxigênio no pulmão do recém-nascido, particularmente no pré-termo devido à imaturidade dos sistemas antioxidantes, e o desenvolvimento da DBP.2,10 Assim como relatado no artigo de Cogo et al.,11 os prematuros que desenvolveram a DBP no presente estudo, utilizaram concentrações de oxigênio mais altas que os não doentes. Porém, nesta pesquisa o valor médio da 
$\mathrm{FiO}_{2}$ foi inferior $(0,47)$ quando comparado ao referido pelos autores $(0,62)$.

Por fim, os dados expostos permitem concluir que a redução dos casos de DBP possui relação direta com a prevenção da prematuridade, que é atualmente um dos desafios da saúde pública. Os resultados sinalizam ainda, que a doença foi relacionada, nesta pesquisa, ao manejo clínico e nutricional do neonato, a concentração de oxigênio superior a 0,45, além da instituição precoce e do uso prolongado da VM. Assim, medidas como a redução no tempo de uso de nutrição parenteral e o início precoce de nutrição enteral podem contribuir na redução dos casos de DBP, auxiliando na prevenção do aparecimento de infecções, incrementando o ganho de peso do prematuro e evitando a permanência prolongada sob VM.

Nesta última, o manejo criterioso na instituição da PIP e concentração de oxigênio resultam em uma

\section{Referências}

1. Tapia JL, Agost D, Alegria A, Standen J, Escobar M, Grandi C, Musante G, Zegarra J, Estay A, Ramírez R, Grupo Colaborativo NEOCOSUR. Bronchopulmonary dysplasia: incidence, risk factors and resource utilization in a population of South American very low birth weight infants. J Pediatr. 2006; 82: 15-20.

2. Cerny L, Torday JS, Rehan VK. Prevention and treatment of bronchopulmonary dysplasia: contemporary status and future outlook. Lung. 2008; 186: 75-89.

3. Cunha GS, Mezzacappa-Filho F, Ribeiro JD. Risk factors for bronchoplmonary dysplasia in very low birth weight newborns treated with mechanical ventilation in the first week of life. J Trop Pediatr. 2005; 51: 334-40.

4. Barros LM, Guinsburg R, Miyoshi MH, Peluzzo AV, Figueira SNA, Kopelman BI. Complacência pulmonar com uma hora de vida e displasia broncopulmonar em recémnascidos prematuros. Rev Bras Saúde Matern Infant. 2007; 7: $387-95$

5. Bhering CA, Mochdece CC, Moreira MEL, Rocco JL, Sant'Anna GM. Bronchopulmonary Dysplasia prediction model for 7-day-old infants. J Pediatr. 2007; 83: 163-70.

6. Gonzaga AD, Figueira BBD, Sousa JMA, Carvalho WB. Tempo de ventilação mecânica e desenvolvimento de displasia broncopulmonar. Rev Assoc Med Bras. 2007; 53: 64-7.

7. Teixeira AB, Xavier CC, Lamounier JA, Tavares EC. Hiperóxia e risco aumentado de displasia broncopulmonar em prematuros. Rev Paul Pediatr. 2007; 25: 47-52.

8. Lima MRO, Andrade MA, Araújo APG, Figueroa JN Andrade LB. Influência dos fatores maternos e neonatais no desenvolvimento da displasia broncopulmonar. Rev Assoc Med Bras. 2011; 57: 398 - 403

9. Gagliardi L, Bellù R, Lista G, Zanini R. Do differences in delivery room intubation explain different rates of bron-
VM “gentil", protegendo o pulmão do prematuro, colaborando para a redução na ocorrência da DBP conforme observado na presente pesquisa.

Futuras pesquisas de caráter prospectivo e com tecnologia que permita análises da função pulmonar, podem fornecer informações úteis sobre as estratégias ventilatórias e de exposição ao oxigênio as quais esta população é submetida, ajudando a estabelecer condutas que minimizem a injúria pulmonar, reduzindo os casos de DBP.

\section{Agradecimentos}

Ao Professor Ricardo Arraes de Alencar Ximenes, do Departamento de Medicina Tropical da Universidade Federal de Pernambuco, pelos comentários e sugestões durante a construção do método e análise dos dados deste artigo.

chopulmonary dysplasia between hospitals? Arch Dis Child Fetal Neonatal Ed. 2010; 96: 30-5.

10. Jobe AH, Bancalari E. Bronchopulmonary dysplasia. Am J Respir Crit Care Med. 2001; 163: 1723-9.

11. Cogo PE, Zimmermann LJ, Pesavento R, Sacchetto E, Burighel A, Rosso F, Badon T, Verlato G, Carnielli VP. Surfactant kinetics in preterm infants on mechanical ventilation who did and did not develop bronchopulmonary dysplasia. Crit Care Med. 2003; 31: 1532-8.

12. Henderson-Smart D J, Hutchinson J L, Donoghue D A, Evans N J, Simpson J M, Wright I. Prenatal predictors of chronic lung disease in very preterm infants. Arch Dis Child Fetal Neonatal Ed. 2006; 91: 40-5.

13. Biniwale MA, Ehrenkranz RA. The role of nutrition in the prevention and management of bronchopulmonary dysplasia. Semin Perinatol. 2006; 30: 200-8.

14. Been JV, Rours IG, Kornelisse RF, Jonkers F, Krijger RR, Zimmermann LJ. Chorioamnionitis alters the response to surfactant in preterm infants. J Pediatr. 2010; 156: 10-5.

15. Van Marter LJ, Allred EN, Pagano M, Sanocka U, Parad R, Moore M, Susser M, Paneth N, Leviton A. Do clinical markers of barotrauma and oxygen toxicity explain interhospital variation in rates of chronic lung disease? Pediatrics. 2000; 105: 1194-201.

16. Srisuparp P, Marks JD, Khoshnood B, Schreiber MD Predictive power of initial severity of pulmonary disease for subsequent development of bronchopulmonary dysplasia. Biol Neonate. 2003; 84: 31-6.

17. Lubchenco LO, Hasman C, Dressler M, Boyd E. Intrauterine growth as estimated from liveborn birth weight data at 24 to 42 weeks of gestation. Pediatrics. 1963; 32: 793 800 .

18. American Academy of Pediatrics, American College of Obstetricians and Gynecologists. The Apgar score. 
Pediatrics. 2006; 117: 1444-7.

19. Jobe AH, Hillman N, Polglase G, Kramer BW, Kallapur S, Pillow J. Injury and inflammation from resuscitation of the preterm infant. Neonatol. 2008; 94: 190-6.

20. Klinger G, Levy I, Sirota L, Boyko V, Lerner-Geva L, Reichman B. Outcome of early-onset sepsis in a national cohort of very low birth weight infants. Pediatrics. 2010; 125: 736-40

21. Mota EM, Garcia PCR, Piva JP, Fritscher CC. A influência da desnutrição na utilização de ventilação mecânica em crianças admitidas em UTI pediátrica. J Pediatr. 2002; 78: 146-52.

22. Duarte JLMB, Mendonça GAS. Fatores associados à morte neonatal em recém-nascidos de muito baixo peso em quatro maternidades no município do Rio de Janeiro, Brasil. Cad Saúde Pública. 2005; 21: 181-91.

23. Latini G, De Felice C, Presta G, Rosati E, Vacca P. Minimal handling and bronchopulmonary dysplasia in extremely low-birth-weight infants. Eur J Pediatr. 2003; 162: 227-9.

24. Turunen R, Nupponen I, Siitonen S, Repo H, Andersson S. Onset of mechanical ventilation is associated with rapid activation of circulating phagocytes in preterm infants
Pediatrics. 2006; 117: 448-54

25. Valenza F, Guglielmi M, Irace M, Porro GA, Sibilla S, Gattinoni L. Positive end-expiratory pressure delays the progression of lung injury during ventilator strategies involving high airway pressure and lung overdistension. Crit Care Med. 2003; 31: 1993-8.

26. Bohrer B, Silveira RC, Neto EC, Procianoy RS. Mechanical ventilation of newborns infant changes in plasma pro- and anti - inflammatory cytokines. J Pediatr. 2010; 156: 16-9.

27. Ventrice EA, Martí-Sistac O, Gonzalvo R, Villagrá A, López-Aguilar J, Blanch L. Mecanismos biofísicos, celulares y modulación de la lesión pulmonar inducida por la ventilación mecánica. Med Intensive. 2007; 31: 73-82.

28. Silva AS, Proença Filho JO. Lesão pulmonar induzida pela ventilação pulmonar mecânica. In: Carvalho WB, Hirschheimer MR, Proença Filho JO, Freddi NA, Troster EJ, editors. Ventilação pulmonar mecânica em pediatria e neonatologia. 2 ed. São Paulo: Atheneu; 2005. p. 535-41.

29. McCallion N, Davis PG, Morley CJ. Ventilación con volumen definido versus ventilación com presión limitada em el neonato. Cochrane Database Syst. Rev. (online). 2007; 4:1-25.

Recebido em 12 de julho de 2011

Versão final apresentada em 12 de março de 2012

Aprovado em 16 de abril de 2012 